\title{
Oral contraceptive use and clinical outcomes in patients with multiple sclerosis
}

\author{
Armando Sena ${ }^{a, *}$, Rémy Couderc ${ }^{b}$, Joana C. Vasconcelos ${ }^{c}$, Véronique Ferret-Sena ${ }^{\mathrm{d}}$, Rui Pedrosa ${ }^{\mathrm{e}}$ \\ a CEDOC Faculty of Medical Sciences, Universidade Nova de Lisboa and Neurology Department, Santo Antonio dos Capuchos Hospital, Lisbon, Portugal \\ b Department of Clinical Chemistry and Molecular Biology, Armand Trousseau University Hospital, Assistance Publique des Hôpitaux de Paris, Paris, France \\ c Department of Clinical Research, BioEpi Clinical and Translational Research Centre, Oeiras, Portugal \\ ${ }^{\mathrm{d}}$ Interdisciplinary Centre of Research Egas Moniz, Cooperativa Egas Moniz, Monte de Caparica, Portugal \\ e Neurology Department, Santo Antonio dos Capuchos Hospital, Lisboa, Portugal
}

\section{A R T I C L E I N F O}

\section{Article history:}

Received 22 November 2011

Received in revised form 5 February 2012

Accepted 28 February 2012

Available online 28 March 2012

\section{Keywords:}

Relapsing-remitting multiple sclerosis (RRMS)

Oral contraceptives

Sex steroids

Neuroprotection

Multiple Sclerosis Severity Score (MSSS)

\begin{abstract}
A B S T R A C T
Experimental and clinical data suggest a role of sex steroids in the pathogenesis of multiple sclerosis (MS). Scant information is available about the potential effect of oral contraceptive (OC) use on the prognosis of the disease. We aimed to evaluate this. The study population consisted of 132 women with relapsing-remitting MS before receiving disease modifying treatment and a mean disease duration 6.2 (SD 5.1) years. Three groups of patients were distinguished according to their OC behavior: [1] never-users, patients who never used OC [2] past-users, patients who stopped OC use before disease onset, and [3] after-users, those who used these drugs after disease onset. Multiple linear and logistic regression models were used to analyze the association between oral contraceptive use and annualized relapse rates, disability accumulation and severity of the disease. After-user patients had lower Expanded Disability Status Scale (EDSS) and Multiple Sclerosis Severity Score (MSSS) values than never users $(p<0.001$ and $p=0.002$, respectively) and past users ( $p=0.010$ and $p=0.002$, respectively). These patients were also more likely to have a benign disease course (MSSS $<2.5$ ) than never and past users together (OR: 4.52, 95\%CI: 2.13-9.56, $p<0.001$ ). This effect remained significant after adjustment for confounders, including smoking and childbirths (OR: 2.97, 95\%CI: $1.24,6.54, p=0.011$ and for MSSS $\beta$ : $-1.04 ; 95 \%$ C.I. $-1.78,-0.30, p=0.006)$. These results suggest that OC use in women with relapsing-remitting MS is possible associated with a milder disabling disease course.
\end{abstract}

(c) 2012 Elsevier B.V. All rights reserved.

\section{Introduction}

Multiple sclerosis (MS) is an inflammatory disease of the central nervous system characterized by demyelization and neurodegeneration. More than $80 \%$ of all MS patients present initially with acute attacks (relapses) of neurological dysfunction, characterizing the relapsing-remitting phenotype (RR-MS) [1]. A large body of evidence suggests that gender influences the susceptibility and the clinical course of the disease. A higher prevalence of women with the RRMS and a female-specific increase in the incidence of the disease has been documented, mainly from an increase in the number of female patients with RR-MS [2]. Gender-related differences in clinical severity, immunological features and brain damage have also been reported, suggesting an effect of sex hormones [3,4]. Moreover, in mice with experimental autoimmune encephalomyelitis (EAE), the animal model of MS, treatment with sex hormones is able to protect against the development of the disease or reduce the severity of its clinical signs [5].

\footnotetext{
* Corresponding author at: Faculdade de Ciências Médicas, Campo Mártires da Pátria, 130, 1169-056 Lisboa, Portugal. Tel.: + 351 218803033; fax: + 351218803010. E-mail address: armando.sena@fcm.unl.pt (A. Sena).
}

Oral contraceptives $(\mathrm{OC})$ constitute a source of exogenous sex hormones for many women. Therefore, OC use may provide an opportunity to assess the influence of these hormones on the risk and clinical course of MS. Some studies have suggested a delay in the age of disease onset (DO) associated with OC use [6,7]. Nevertheless, despite its practical interest for MS women who decide to take this preventive method of conception, only few inconclusive studies have addressed the impact of its use on the prognosis of the disease $[8,9]$. These studies are complicated by the influences of potential confounders and by modification of the effect of OC use from differential treatment effects ("interaction bias"). Most patients with RR-MS are now treated very early with immunomodulatory agents, which could alter the natural history of the disease, though some may not respond or have poor responses to these treatments [10]. In order to investigate a potential association between OC use and the clinical course of the disease it would be important to avoid interaction bias that heterogeneous responses to current therapies could create. The present retrospective and exploratory study was designed to examine the cross-sectional association of OC use with the clinical course of RR-MS patients before they initiated disease-modifying therapies. The association with possible confounders including smoking habits and childbearing was also investigated. In a subset of patients we further analyzed the influence of OC use on serum levels of vitamin D. 


\section{Methods}

\subsection{Patients and data collection}

The studied population belong to a cohort of 145 women with RRMS according to the revised McDonald criteria [11], followed at the MS outpatient clinic (Neurology Department) of a central University Hospital in Lisbon, Portugal. The study included patients first examined between 1995 and 2010 with at least 2 years duration of disease. Clinical and demographic information was collected from patient notes at the visit to the neurologist prior to initiation of immunomodulatory or immunosuppressive treatments. No patients had been treated with steroids for at least 1 month. The clinical neurological data that were collected comprised the age at onset of MS, disease duration, annualized relapse rate and disease disability and severity, assessed by using the Expanded Disability Status Scale (EDSS) [12] and the Multiple Sclerosis Severity Score (MSSS) [13], respectively. The MSSS is based on EDSS score adjusted for disease duration and is currently the best method to compare the severity of disease course between patients [13,14]. MSSS scores were obtained from Fig. 3 of the paper by Roxburg et al. [13]. The MSSS values were dichotomized using a cut-off of MSSS $<2.5$ to allow comparison of relatively benign patients with more severely affected patients [14]. The age at disease onset (DO) was defined as the earliest date at which neurological symptoms attributed to MS were reported. A relapse was defined as the appearance or worsening of a neurological disturbance lasting over 24 hours and followed by a variable degree of recovery [1]. The smoking questionnaire contained questions on current smoking status, starting and stopping dates and number of cigarettes smoked. Smokers were defined based on the report of smoking at least five cigarettes per day. Gynecological and obstetric histories were obtained from every patient and included age at menarche, age at initiation of OC use, duration of OC use, dates and number of full-term deliveries (parity), dates of menopause and gynecological surgical interventions. Women who never used OC were categorized as "neverusers". Women using the pill after DO were classified as after-users and those who discontinued its use before DO were defined as pastusers. We defined a patient to be an OC user when the prescription was adhered to for a continuous period of at least 12 months. We were unable to take the composition of the prescribed pill into account, because this information was lacking for some patients and many changed the type or brand of the drug. The most frequently used pills were combinations of an oestrogen (usually ethinyl oestradiol) and a progestogen. Approval for the study by the local Medical Ethical Committee was obtained and all patients gave written informed consent.

\subsection{Measurement of vitamin $D$}

Serum 25-hydroxyvitamin D (25(OH)VD) levels and body mass index (BMI) were measured in 37 patients (17 current-users and 20 never-users) and 32 age-matched female normal controls (17 current-users and 15 never-users). All serum samples were stored at $-80{ }^{\circ} \mathrm{C}$ and measurement of $25(\mathrm{OH}) \mathrm{VD}$ performed in blinded conditions regarding subject participants. Vitamin D was measured by Dia Sorin LIAISON technique which uses an antibody against 25-OH-D in a 2-site chemiluminescence assay.

\subsection{Statistical analysis}

Means (standard deviations (SD)) and medians (inter-quartile ranges (IQR)) were used to describe demographic and clinical data for each OC use behavior (never-users, past-users and after-users). Data were pairwise-compared among these groups using two-sided Mann-Whitney non-parametric tests for continuous data or Fisher's Exact tests for categorical variables. Multiple linear regression was used to assess the effect of OC use on the main clinical variables (EDSS, MSSS and relapse rates) with adjustment for possible confounders. Confounders taken into account were age at DO, age, disease duration, smoking status, and age at menarche.

Demographic and clinical data were pairwise-compared among the three types of $\mathrm{OC}$ users in the subgroup of patients with an MSSS $<2.5$ using the Mann-Whitney or Fisher's Exact tests. The effect of OC use (after-users versus never and past-users) on the prediction of a benign course of disease (MSSS $<2.5$ ) was analyzed using logistic regression to evaluate its effect after adjusting for confounders. The effect of OC use was assessed on the MSSS values using multiple linear regression to adjust for parity and potential confounders. The OC effect was also estimated amongst women with children, adjusting for the number of children and the timing of children relative to DO.

The effect of OC use on age at DO was assessed using multiple linear regression, adjusting for potential confounders, and the effect of OC duration on age at DO was evaluated using the non-parametric test for trend, after OC duration was categorized into 1 to 3 years, 4 to 5, 6 to 10, and more than 10 years of OC duration [7]. Vitamin D levels were compared between groups by analysis of variance (ANOVA).

Statistical significance was considered at the 5\% level and 95\% confidence intervals were computed for odds ratios (OR) and beta coefficients. All analyses were carried out using the statistical software STATA v10.0.

\section{Results}

From the cohort of 145 RR-MS patients, those in menopause $(n=7)$ or who underwent gynecological surgeries $(n=6)$ were excluded, leaving 132 women for the analysis. Among these, 52 were never-users, 26 were past-users and 54 were after-users. All but nine of the after-user patients started the prescription before DO. A summary of the demographic and clinical characteristics of these patients, stratified according to OC use, are presented and compared in Table 1 . EDSS and MSSS values were significantly lower in afterusers than past-users ( $p=0.015$ for EDSS and $p=0.002$ for MSSS) or never-users $(p<0.001$ for EDSS and $p=0.002$ for MSSS). For the comparison between never-user and after-users, adjustment for age at DO, disease duration, smoking status and age at menarche left the significance unchanged for the EDSS and MSSS $(\beta=-0.69,95 \%$ C.I. $-1,13,-0.24, p=0.003$ for EDSS and $\beta=-1.29,95 \%$ C.I. -2 , $12,-0.47, p=0.003$ for MSSS). No association between OC behavior and the annualized relapse rates was seen even when adjusting for these confounders ( $\beta=-0.08,95 \%$ C.I. $-0,29,0.14, p=0.481)$.

Among the patients with a more benign disease course (patients with an MSSS $<2.5$ ) (Table 2) no association was found between OC use and age, age at DO, disease duration and relapse rate. A significantly higher number of after-users (59\%) in comparison to never and past-users $(24 \%)$ were observed in this subset of patients $(p<0.001)$. Never-users were joined with past-users because only six patients had a benign course or had no children in the pastusers group. Logistic regression (Table 3 ) showed that patients who took OC after DO were more likely to have a more benign course than never and past-users (OR: 4.52; 95\% CI: 2.13, 9.56, $p<0.001$ ). This protective effect of $\mathrm{OC}$ intake remained sizeable and significant even after adjusting for potential confounders (Table 3: OR 2.97, 95\% C.I. 1.24, 6.54, $p=0.011$ and Table 4: $\beta=-1.04,95 \%$ C.I. $-1.78,-0.30, p=0.006)$. Age at DO and age were too highly correlated to adjust for both, though the OC intake effect remained significant when age was adjusted for instead of age at DO $(p=0.014$ and $p=0.007$, for the equivalent in Tables 3 and 4, respectively).

Parity was not associated with OC use (Table 1), nor was associated with a benign course of disease, over and above the effect of OC and confounders (Table 3: OR 0.52 95\% CI 0.19, 1.41, $p=0.202$ ). No 
Table 1

Comparison of demographic and clinical characteristics by patient's oral contraceptive use behavior.

\begin{tabular}{|c|c|c|c|c|c|c|c|c|c|}
\hline & \multicolumn{2}{|l|}{$\begin{array}{l}\text { Never-users } \\
(n=52)\end{array}$} & \multicolumn{2}{|l|}{$\begin{array}{l}\text { Past-users } \\
(n=26)\end{array}$} & \multirow[t]{2}{*}{$\begin{array}{l}p- \\
\text { Value }\end{array}$} & \multicolumn{2}{|l|}{$\begin{array}{l}\text { After-users } \\
(n=54)\end{array}$} & \multirow{2}{*}{$\begin{array}{l}p \text {-Value for } \\
\text { comparison } \\
\text { with Never }\end{array}$} & \multirow{2}{*}{$\begin{array}{l}p \text {-Value for } \\
\text { comparison } \\
\text { with Past }\end{array}$} \\
\hline & Mean (SD) & Median (IQR) & Mean (SD) & Median (IQR) & & Mean (SD) & Median (IQR) & & \\
\hline Age & $37.3(10.2)$ & $39.0(31.0,44.0)$ & $38.6(6.8)$ & $39.0(34.0,43.0)$ & 0.795 & $32.0(6.6)$ & $32.0(26.0,36.0)$ & $<0.001$ & $<0.001$ \\
\hline Age at disease onset & $29.9(9.8)$ & $30.5(21.0,37.5)$ & $34.6(6.9)$ & $36.0(29.0,41.0)$ & 0.021 & $25.8(6.1)$ & $25.0(21.0,30.0)$ & 0.018 & $<0.001$ \\
\hline Disease duration & $7.2(6.1)$ & $5.0(2.0,10.0)$ & $4.0(2.6)$ & $3.0(2.0,6.0)$ & 0.040 & $6.2(4.6)$ & $5.0(3.0,9.0)$ & 0.776 & 0.052 \\
\hline Relapse rate & $0.99(0.57)$ & $1.0(0.5,1.0)$ & $1.02(0.66)$ & $1.0(0.5,1.5)$ & 0.823 & $0.89(0.52)$ & $1.0(0.5,1.0)$ & 0.284 & 0.584 \\
\hline EDSS & $2.55(1.30)$ & $2.5(1.8,3.5)$ & $2.31(1.34)$ & $2.0(2.0,3.5)$ & 0.416 & $1.60(1.03)$ & $1.5(1.0,2.0)$ & $<0.001$ & 0.015 \\
\hline MSSS & $4.17(2.46)$ & $3.8(2.5,5.9)$ & $4.64(2.53)$ & $4.9(3.2,6.5)$ & 0.382 & $2.69(1.87)$ & $2.2(1.3,4.1)$ & 0.002 & 0.002 \\
\hline Age of menarche & $12.6(1.6)$ & $12.5(11.5,14.0)$ & $11.8(1.6)$ & $12.0(11.0,12.0)$ & 0.022 & $11.9(1.6)$ & $12.0(11.0,13.0)$ & 0.043 & 0.482 \\
\hline Age at onset of $O C$ use & $\mathrm{N} / \mathrm{A}$ & & $21.1(3.7)$ & $20.5(18.0,23.0)$ & $\mathrm{N} / \mathrm{A}$ & $20.1(4.0)$ & $19.0(18.0,22.0)$ & $\mathrm{N} / \mathrm{A}$ & 0.172 \\
\hline OC use duration & $\mathrm{N} / \mathrm{A}$ & & $7.6(5.9)$ & $5.5(3.0,11.0)$ & $\mathrm{N} / \mathrm{A}$ & $10.5(5.8)$ & $10.5(6.0,15.0)$ & $\mathrm{N} / \mathrm{A}$ & 0.022 \\
\hline Ever smoker $n(\%)$ & $15(29 \%)$ & & $13(50 \%)$ & & 0.083 & $22(41 \%)$ & & 0.226 & 0.477 \\
\hline Parous $n(\%)$ & $36(69 \%)$ & & $20(77 \%)$ & & 0.597 & $31(57 \%)$ & & 0.232 & 0.136 \\
\hline Number of children & $\begin{array}{l}1.6(0.7) \\
n=36\end{array}$ & $\begin{array}{l}1.5(1.0,2.0) \\
n=36\end{array}$ & $\begin{array}{l}1.8(0.5) \\
n=20\end{array}$ & $\begin{array}{l}2.0(1.5,2.0) \\
n=20\end{array}$ & 0.144 & $\begin{array}{l}1.6(0.7) \\
(n=31)\end{array}$ & $\begin{array}{l}2.0(1.0,2.0) \\
(n=31)\end{array}$ & 0.791 & 0.278 \\
\hline Age at 1 st child & $\begin{array}{l}25.1(4.8) \\
n=36\end{array}$ & $\begin{array}{l}25.0(21.0,28.5) \\
n=36\end{array}$ & $\begin{array}{l}23.1(3.5) \\
n=20\end{array}$ & $\begin{array}{l}23.5(20.0,25.5) \\
n=20\end{array}$ & 0.101 & $\begin{array}{l}23.9(5.1) \\
(n=31)\end{array}$ & $\begin{array}{l}24.0(20.0,27.0) \\
(n=31)\end{array}$ & 0.281 & 0.587 \\
\hline
\end{tabular}

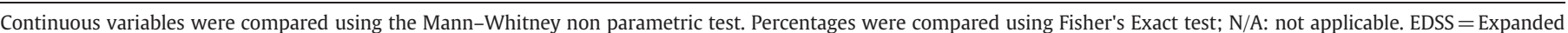
Disability Status Scale MSSS = Multiple Sclerosis Severity Score.

Table 2

Comparison of patients' characteristics with a benign course of disease $(\mathrm{MSSS}<2.5$ ) by patients' oral contraceptive use behavior.

\begin{tabular}{|c|c|c|c|c|c|}
\hline & \multicolumn{2}{|c|}{$\begin{array}{l}\text { Never and past users } \\
(n=19)\end{array}$} & \multicolumn{2}{|l|}{$\begin{array}{l}\text { After users } \\
(n=32)\end{array}$} & \multirow[t]{2}{*}{$p$-Value } \\
\hline & Mean (SD) & Median (IQR) & Mean (SD) & Median (IQR) & \\
\hline$\%$ of total & $24 \%(19 / 78)$ & & $59 \%(32 / 54)$ & & $<0.001$ \\
\hline Age & $30.0(8.9)$ & $30.0(25.0,38.0)$ & $31.5(5.8)$ & $31.0(27.5,35.5)$ & 0.689 \\
\hline Age at disease onset & $23.9(7.7)$ & $23.0(18.0,30.0)$ & $25.0(6.0)$ & $24.5(20.5,28.0)$ & 0.564 \\
\hline Disease duration & $6.1(6.5)$ & $3.0(2.0,7.0)$ & $6.4(4.3)$ & $5.0(3.0,10.0)$ & 0.177 \\
\hline Relapse rate & $0.84(0.59)$ & $0.5(0.5,1.0)$ & $0.83(0.56)$ & $0.75(0.5,1.0)$ & 0.967 \\
\hline Parous $n(\%)$ & $8(42 \%)$ & & $16(50 \%)$ & & 0.772 \\
\hline
\end{tabular}

Continuous variables were compared using Mann-Whitney non-parametric test. Percentages were compared using Fisher's Exact test.

interaction between OC use and parity on MSSS values was found, with parity having no significant effect on MSSS (Table 4: $\beta=0.77$; $95 \% \mathrm{CI}:-0.11,1.64, p=0.085)$. Among women with children, having the children after as opposed to only before DO, or having a high number of children, were not associated with MSSS after accounting for the effect of $O C$ and confounders (Table 4).

Having children before DO $(\beta=5.18$; $95 \% \mathrm{CI}: 3.12,7.23, p<0.001)$, and using OC before DO ( $\beta=1.99 ; 95 \% \mathrm{CI}: 0.30,3.69, p=0.022)$ were associated with a later age at DO when adjusting for age. The effect of OC on age at DO was not significant when adjusting for parity and age $(\beta=1.49 ; 95 \% \mathrm{CI}:-0.14,3.12, p=0.073)$, with parity having a significant effect independent of these other factors $(\beta=3.96 ; 95 \% \mathrm{Cl}: 1.85$, 6.06, $p<0.001$ ). In the subgroup of patients who took OC before DO $(n=71)$, a higher OC intake duration appeared to be related to a later age of DO $(\beta=0.50 ; 95 \% \mathrm{Cl}: 0.24,0.76, p<0.001)$. Having categorized OC durations into 1 to 3 years, 4 to 5,6 to 10 and more than 10 years of OC intake, the mean (SDs) of age at DO were 26.3 (6.3), 28.1 (6.7), 29.5 (8.3) and $31.6(6.6)$, respectively (test for trend $p=0.026)$.

Patients had lower mean 25(OD)VD serum levels than female normal controls (mean 18.9 (SD 8.9) vs. 25.5 (SD 11.8) ng/mL, $p=0.012$ ). No significant differences were found between women who were OC users in comparison to never-users in patients and controls. In addition, no significant associations were found between vitamin D levels and smoking status, BMI values and age of subjects in our study population.

\section{Discussion}

Classifications based on the MSSS have been considered the best available methods for severity assessment of MS and discrimination of benign forms from more rapidly disabling disease courses $[13,14]$. Therefore, our results suggest that women who use OC after the first symptoms of the disease have a milder disease course in comparison to those who never used OC or stopped its intake before DO. As previously suggested, we found that $O C$ use and having children before DO [6,7] as well as OC intake duration [7] might be associated with a delayed age at DO. Nevertheless, the protective effect on disability accumulation observed when OC use was maintained after the appearance of the first symptoms of the disease was independent of age, age at DO, OC intake duration and other studied potential confounders, including smoking habits and parity.

Table 3

Effect of oral contraceptive use after disease onset and clinical characteristics on predicting a benign course of disease.

\begin{tabular}{llc}
\hline Variables & Odds ratio (95\% C.I.) & $p$-Value \\
\hline $\begin{array}{l}\text { Univariate analysis }(n=132): \text { OC effect } \\
\quad \text { (after users vs never and past users) }\end{array}$ & $4.52(2.13,9.56)$ & $<0.001$ \\
Multivariate analysis ${ }^{\mathrm{a}}(n=132)$ & & \\
OC effect (after users vs never and past users) & $2.97(1.24,6.54)$ & 0.011 \\
Age at disease onset & $0.91(0.85,0.97)$ & 0.005 \\
Disease duration & $0.97(0.89,1.06)$ & 0.541 \\
Relapse rate & $0.55(0.24,1.29)$ & 0.170 \\
Smoker & $1.69(0.69,4.10)$ & 0.248 \\
Age of menarche & $1.01(0.77,1.33)$ & 0.935 \\
Parous (vs nulliparous) & $0.52(0.19,1.41)$ & 0.202 \\
\hline
\end{tabular}

$\mathrm{OC}=$ oral contraceptives.

a In the multivariate analysis the effects of OC and clinical characteristics on MSSS were adjusted for each other. Age was not included because it is highly correlated with age at disease onset. 
Table 4

Multiple linear regression models to determine the effect of oral contraceptive use and parity on the Multiple Sclerosis Severity Score.

\begin{tabular}{|c|c|c|c|c|c|}
\hline \multirow[t]{2}{*}{ Factors: } & \multicolumn{2}{|l|}{$\begin{array}{l}\text { All patients } \\
n=132\end{array}$} & \multirow[t]{2}{*}{ Factors: } & \multicolumn{2}{|l|}{$\begin{array}{l}\text { Women with children } \\
n=87\end{array}$} \\
\hline & $\beta(95 \% \text { C.I. })^{*}$ & $p$-Value & & $\beta(95 \% \text { C.I. })^{*}$ & $p$-Value \\
\hline After-users (vs never and past users) & $-1.04(-1.78,-0.30)$ & 0.006 & After-users (vs never and past users) & $-1.15(-2.11,-0.20)$ & 0.018 \\
\hline \multirow[t]{2}{*}{ Parous (vs nulliparous) } & $0.77(-0.11,1.64)$ & 0.085 & Children after DO (vs children only before DO) & $-0.53(-1.72,0.67)$ & 0.383 \\
\hline & & & No. children (1, 2 or more) & $-0.24(-1.10,0.62)$ & 0.580 \\
\hline
\end{tabular}

* Adjusted for age at disease onset, disease duration, annual relapse rate, ever smoking and menarche.

The influence of smoking on the progression of MS is still controversial $[15,16]$ and results from one study suggested gender-related differences in the association of pack-years smoked with measures of accumulation disability [15]. Our study indicates that intake of OC could have been a potential confounder in these studies. An influence of pregnancies or childbirths on disability progression of MS has been found by some [17] but not all studies [18,19]. Differences in methodology and patient sample may account for this discrepancy. Most importantly, an OC behavior bias cannot be removed from these studies. Our data do not support any independent influence of parity and number of childbirths on MSSS outcomes when adjusting for OC use.

No influence of OC use on relapse rates was found in this study. It is known that relapse frequency does not directly influence the rate of sustained disability in these patients [20]. Increasing evidence indicates that biological mechanisms leading to disability progression implicate a neurodegenerative process and may differ from those responsible for acute relapses [21,22]. Our results could thus suggest a promotion of repairing and neuroprotective mechanisms associated with OC use. This interpretation is supported by experimental and clinical data indicating that sex hormones may have protective effects on brain damage and repair mechanisms in these patients [3-5]. All oestrogens are not exactly alike [5,23]. Importantly and unlike $17 \beta-$ oestradiol, the common oestrogen found in pills ethynil oestradiol $[24,25]$ and progesterone [26] reduce the clinical severity of EAE when given after the onset of disease signs. Because the large majority of our patients have used different combinations of ethynil oestradiol and a progestogen, we cannot exclude that these hormones could have different impacts on the observed protective effects. Future work should address the potential of influence of progestogen-only pills on the clinical course of the disease. Oestrogens and vitamin $\mathrm{D}$ have mutual metabolic relationships [27] and an insufficiency of vitamin D have been implicated as a risk factor for MS [28]. In these patients, a recent study has found that lower levels of 25(OH)VD were associated with higher MSSS [29]. Most patients in this study were receiving disease modifying treatment and no information regarding OC use was provided. We did not observed significant variations of $25(\mathrm{OH}) \mathrm{VD}$ with OC use. However, we should be cautious with the interpretation of these results because of the small cohort of women analyzed and potential bias, including sun exposure, seasonality and diet $[28,29]$. Further studies investigating the association of OC use with vitamin D metabolites are warranted. Current immunologic treatments for RR-MS are known to have a modest effect in preventing accumulation of disability [22]. While safety concerns associated with long-term therapeutic use of sex hormones should be considered [5] our results support a potential benefice of such compounds or their derivatives in combination with currently available immunomodulatory agents for the disease [23]. Overall, our results and previous work suggest that MS women considering the use of OC should not base their decision on potential deleterious effects on their clinical course. Rather, our data suggest a possible beneficial effect.

Our study has several limitations. It was limited in being a single center study, although in a generalizable clinic-based population. Data were collected retrospectively, though from well-kept patient records from a cohort of patients that was well-defined crosssectionally according to the time of disease onset. As with any observational study, the suggested association of OC use with a milder disease does not ascertain a causal relationship. Because after-users were younger than past and never-users patients, we cannot exclude a partial confounding effect of age. However, multivariate analysis was undertaken to control for this issue and the OC effect remained significant after adjusting for age. Moreover, our study is based on a sample population of RR-MS patients with a short duration of the disease and so we cannot infer from these data a favorable long-term effect of OC use in latter stages of the disease. Nevertheless, the clinical course of MS is highly variable and some patients develop high disability within a short period of time. The confirmation that OC use may delay disability accumulation early in the course of the disease could be of considerable interest. It is increasingly impractical and unethical to carry out a study on a large population of RR-MS patients with long disease duration and devoid of disease-modifying therapies. As discussed above, our findings need to be confirmed in prospective long-term follow-up studies in patients medicated with current immunomodulatory agents. Information relating tissue damage with OC behavior was not collected and should be assessed by MRI in future studies. Disease severity in MS is thought to be determined by environmental as well as genetic factors [14]. Although genetic data were not included in our study, the reported influence of these factors on disability progression has been modest and controversial [14]. Finally, it is possible that the type or composition of pills might be relevant for their potential benefits in the disease. Future research needs to clarify this issue, which could be of practical importance for MS women who decide to take this preventive method of conception and to design sex-hormone based therapies for this disease.

\section{Funding}

This work was supported by Bayer Health Care (PILLBETA project) and Biogen Idec, in Portugal.

\section{Conflict of interest statement}

Armando Sena has received personal compensation for consulting or speaking from Merck Serono and Biogen Idec and financial support for research activities from Merck Serono, Biogen Idec, Bayer Health Care and Sanofi-Aventis. Rui Pedrosa has received personal compensation as an advisor board member of Novartis and for consulting or speaking from Merck Serono, Bayer Health Care and Biogen Idec. Remy Couderc, Joana C Vasconcelos and Véronique Ferret-Sena have nothing to disclose.

\section{Acknowledgements}

The authors thank to nurses Cristina Araújo and Ana Mendes (Neurology Service) for help in research and to Dr Elizabeth BenitoGarcia (BioEPI, Clinical and Translational Research Centre) for epidemiological revision of the manuscript. 


\section{References}

[1] Lublin FD, Reingold SC. Defining the clinical course of multiple sclerosis: results of an international survey. National Multiple Sclerosis Society (USA) Advisory Committee on Trials of New Agents in Multiple Sclerosis. Neurology 1996;46:907-11.

[2] Ramagopalan SV, Byrnes JK, Orton S-M, Dyment DA, Guimond C, Yee IM. Sex ratio of multiple sclerosis and clinical phenotype. Eur J Neurol 2010;17:634-7.

[3] Tomassini V, Pozzilli C. Sex hormones, brain damage and clinical course of multiple sclerosis. J Neurol Sci 2009;286:35-9.

[4] Antulov R, Weinstock-Guttman B, Cox JL, Hussein S, Durfee J, Caiola C, et al. Gender-related differences in MS: a study of conventional and nonconventional MR measures. Mult Scler 2009;15:345-54.

[5] Gold SM, Voskuhl RR. Estrogen treatment in multiple sclerosis. J Neurol Sci 2009;286:99-103.

[6] Alonso A, Jick SS, Olek MJ, Ascherio A, Jick H, Hernán MA. Recent use of oral contraceptives and the risk of multiple sclerosis. Arch Neurol 2005;62:1362-5.

[7] Holmqvist P, Hammar M, Landtblom A-M, Brynhildsen J. Age at onset of multiple sclerosis is correlated to use of combined oral contraceptives and childbirth before diagnosis. Fertil Steril 2010;94:2835-7.

[8] Poser S, Raun NE, Wikstrom J, Poser W. Pregnancy, oral contraceptives and multiple sclerosis. Acta Neurol Scand 1979;59:108-1189.

[9] Holmqvist P, Wallberg M, Hamman M, Landtblom A-M, Brynhildsen J. Symptoms of multiple sclerosis in women in relation to sex steroid exposure. Maturitas 2006;54:149-53.

[10] Comabella M, Lunemann JD, Rio J, Sanchez A, Lopez C, Julia E, et al. A type I interferon signature in monocytes is associated with poor response to interferon- $\beta$ in multiple sclerosis. Brain 2009;132:3353-65.

[11] Polman CH, Reingold SC, Edan G, Filippi M, Hartung H-P, Kappos L, et al. Diagnostic criteria for multiple sclerosis: 2005 Revisions to the "Mc Donald Criteria». Ann Neurol 2005;58:840-6.

[12] Kurtzke JF. Rating neurologic impairment in multiple sclerosis: an expanded disability status scale (EDSS). Neurology 1983;33:1444-52.

[13] Roxburgh RH, Seaman SR, Masterman T, Hensiek AE, Sawcer SJ, Vukusic S, et al Multiple sclerosis severity score. Using disability and disease duration to rate disease severity. Neurology 2000;64:1144-51.

[14] Sombekke MH, Arteta D, van de Wiel MA, Crusius JBA, Tejedor D, Killestein J. Analysis of multiple candidate genes in association with phenotypes of multiple sclerosis. Mult Scler 2010;16:652-9.

[15] Koch M, Uyttenboogaart A, De Keyser J. Cigarette smoking and progression in multiple sclerosis. Neurology 2007;69:1515-20.
[16] Sena A, Couderc R, Ferret-Sena V, Pedrosa R, Andrade ML, Araujo C, et al. Apolipoprotein E polymorphism interacts with cigarette smoking in progression of multiple sclerosis. Eur J Neurol 2009;16:832-7.

[17] D'hooghe MB, Nagels G, Uitgehaag BMJ. Long-term effects of childbirth in MS. J Neurol Neurosurg Psychiatry 2010;81:38-41.

[18] Damek DM, Schoster EA. Pregnancy and multiple sclerosis. Mayo Clin Proc 1997;72:977-89.

[19] Vukusic S, Hutchinson M, Hours M, Moreau T, Cortinovis-Tourniaire P, Adeleine P, et al. Pregnancy and multiple sclerosis (the PRIMS study): clinical predictors of post-partum relapse. Brain 2004;127:1353-60.

[20] Scalfari A, Neuhaus A, Degenhardt A, Rice GP, Muraro PA, Daumer M, et al. The natural history of multiple sclerosis, a geographically based study 10: relapses and long-term disability. Brain 2010;133:1914-29.

[21] Trapp BD, Nave KA. Multiple sclerosis: an immune or neurodegenerative disorder? Annu Rev Neurosci 2008;31:247-69.

[22] Van der Walt A, Butzkueven H, Kolbe S, Marriott M, Alexandrou E, Gresle M. Neuroprotection in multiple sclerosis: a therapeutic challenge for the next decade. Pharmacol Ther 2010;126:82-93.

[23] Crawford DK, Mangiardi M, Song B, Patel R, Du S, Sofroniew MV, et al. Oestrogen receptor $\beta$ ligand: a novel treatment to enhance endogenous functional remyelination. Brain 2010;133:2999-3016.

[24] Subramanian S, Matejuk A, Zamora A, Vandenbark AA, Offner H. Oral feeding with ethinyl estradiol suppresses and treats experimental autoimmune encephalomyelitis in SJL mice and inhibits the recruitment of inflammatory cells into the central nervous system. J Immunol 2003;170:1548-55.

[25] Yates MA, Li Y, Chlebeck P, Offner H. GPR30, but not estrogen receptor- $\alpha$, is crucial in the treatment of experimental autoimmune encephalomyelitis by oral ethinyl estradiol. BMC Immunol 2010;11:11-20.

[26] Yates MA, Li Y, Chlebeck P, Proctor T, Vandenbark AA, Offner $H$. Progesterone treatment reduces disease severity and increases IL-10 in experimental autoimmune encephalomyelitis. J Neuroimmunol 2010;220:136-9.

[27] Nashold F, Spach KM, Spanier JA, Hayes CE. (Estrogen controls vitamin D3mediated resitence to experimental autoimmune encephalomyelitis by controlling vitamin D3 metabolism and receptor expression. J Immunol 2009;183: 3672-81.

[28] Mowry EM. Vitamin D: evidence for its role as a prognosis factor in multiple sclerosis. J Neurol Sci 2011;311:19-22.

[29] Weinstock-Guttman B, Zivadinov R, Qu J, Cookfair D, Duan X, Bang E, et al. Vitamin D metabolites are associated with clinical and MRI outcomes in multiple sclerosis patients. J Neurol Neurosurg Psychiatry 2011;82:189-95. 